\title{
Sample Preparation Methodologies for In Situ Liquid and Gaseous Cell Analytical Transmission Electron Microscopy of Electropolished Specimens
} DOI:

10.1017/S1431927616011855

\section{Document Version \\ Accepted author manuscript}

Link to publication record in Manchester Research Explorer

Citation for published version (APA):

Zhong, X., Schilling, S., Zaluzec, N. J., \& Burke, M. G. (2016). Sample Preparation Methodologies for In Situ Liquid and Gaseous Cell Analytical Transmission Electron Microscopy of Electropolished Specimens. Microscopy and Microanalysis, 22(6). https://doi.org/10.1017/S1431927616011855

Published in:

Microscopy and Microanalysis

\section{Citing this paper}

Please note that where the full-text provided on Manchester Research Explorer is the Author Accepted Manuscript or Proof version this may differ from the final Published version. If citing, it is advised that you check and use the publisher's definitive version.

\section{General rights}

Copyright and moral rights for the publications made accessible in the Research Explorer are retained by the authors and/or other copyright owners and it is a condition of accessing publications that users recognise and abide by the legal requirements associated with these rights.

\section{Takedown policy}

If you believe that this document breaches copyright please refer to the University of Manchester's Takedown Procedures [http://man.ac.uk/04Y6Bo] or contact uml.scholarlycommunications@manchester.ac.uk providing relevant details, so we can investigate your claim.

\section{OPEN ACCESS}


North American Branch

32 Avenue of the Americas

New York, NY 10013-2473, USA

\section{PROOFREADING INSTRUCTIONS}

E-mail: jchang@cambridge.org

Dear Microscopy and Microanalysis Contributor:

Attached is a PDF page proof of your introduction/article/book review scheduled to be published in:

\section{Microscopy and Microanalysis}

Please follow these procedures:

1. Proofreading: Proofread your article carefully. Check especially the spellings of names and places as well as the accuracy of dates and numbers. Please answer all queries that may appear on a separate page.

2. Text: Changes in the text are limited to typographical and factual errors. Rewriting or other stylistic changes are not permitted. Contributors may be charged for excessive author alterations, and publication of your article may be delayed.

3. Corrections: Please respond with an e-mail message to Julia Chang (jchang@cambridge.org), identifying the correction by page number, column, paragraph, and line. Please indicate the present errant copy followed by the correct copy. The corrections to the proofs should be sent within 2 days of receipt. Corrections can also be entered into the PDF as embedded comments.

4. References: If the query involves a reference entry, please arrange the new entry into the correct format. In order to prepare the HTML full-text files for online viewing, the complete information including correct spelling of author names, year, titles, publisher, city of publication, page range, and so forth are needed.

5. Figures: Review the figure reproductions on the page proofs to see if important features have been well represented. If something seems out of order, indicate the errant features in a cover letter. Should it be necessary that new electronic copy of the figures (in PDF, TIFF, or EPS) or text (Word or LaTeX) will have to be provided, please indicate which application is being used.

6. Offprints or Bound Copies: Free offprints are longer being offered. However, a message with a link to access a free PDF of your paper will be sent to you. To order reprints or offprints of your article or printed copies of the issue, please visit the Cambridge University Reprint Order Center online at: www.sheridan.com/cup/eoc

7. Delay in response: Please note that failure to respond in a timely fashion may delay publication of your article or may require publication without your corrections. Thank you for your prompt attention to these proofs. If you have any questions, please feel free to contact Julia Chang by e-mail: jchang@cambridge.org. Thanks.

Best Regards, Julia Chang,

Production Editor 


\section{QUERY FORM}

\begin{tabular}{|l|c|}
\hline \multicolumn{2}{|c|}{ MAM } \\
\hline Manuscript ID & [Art. Id: 1601185] \\
\hline Author & \\
\hline Editor & \\
\hline Publisher & \\
\hline
\end{tabular}

\section{Journal: Microscopy And Microanalysis}

Author :- The following queries have arisen during the editing of your manuscript. Please answer queries by making the requisite corrections at the appropriate positions in the text.

\begin{tabular}{|c|c|}
\hline Query No & Nature of Query \\
\hline Q1 & $\begin{array}{l}\text { The distinction between surnames can be ambiguous, therefore to ensure accurate tagging for indexing purposes } \\
\text { online (e.g. for PubMed entries), please check that the highlighted surnames have been correctly identified, that all } \\
\text { names are in the correct order and spelt correctly. }\end{array}$ \\
\hline Q2 & Please limit the key words to a maximum of five key words. \\
\hline Q3 & Please mention whether it is Radisic et al. (2006a) or (2006b) here and in other places in the text. \\
\hline Q4 & $\begin{array}{l}\text { Schilling et al. (2015), Radisic et al. (2009), Matteson et al. (2002) are cited in the text but not listed in the Ref. list, } \\
\text { please provide complete publication details to include in the Ref. list. }\end{array}$ \\
\hline Q5 & $\begin{array}{l}\text { Please provide city/state and country location of the manufacturers 'Protochips Inc.', 'Oxford Instrument', } \\
\text { 'Kleindiek Nanotechnik'. }\end{array}$ \\
\hline Q6 & $\begin{array}{l}\text { Please provide manufacturer details (name of the manufacturer, city/state and country location) for "Struers } \\
\text { Accutom-5 sectioning system", "FEI Quanta 3D Dual-Beam FIB/SEM", "FEI Tecnai T20 Analytical Electron } \\
\text { Microscope", "X-max } 80 \text { TLE windowless Si drift detector", "AZTEC analyzer", "Protochips Poseidon P210 } \\
\text { liquid E-Cell", "Struers TenuPol-5 twin-jet electropolishing unit" and "Jubalo closed cycle refrigeration system". }\end{array}$ \\
\hline Q7 & $\begin{array}{l}\text { Young et al. (2010) has been changed to Young et al. (2008) so that this citation matches the reference list. Please } \\
\text { confirm that this is correct. }\end{array}$ \\
\hline Q8 & The sentence "With this in mind, it should be realized that it will be unavoidable..." is not clear, please check. \\
\hline Q9 & Please check whether the hierarchy followed for section-level headings is correct. \\
\hline Q10 & Please provide city/location of 'Henkel'. \\
\hline Q11 & The sentence "When mounting this holder into the dual-beam FIB..." is not clear, please check. \\
\hline Q12 & $\begin{array}{l}\text { Ziemniak et al. (2002) has been changed to Ziemniak \& Hanson (2002) so that this citation matches the reference } \\
\text { list. Please confirm that this is correct. }\end{array}$ \\
\hline
\end{tabular}

\section{Cambridge University Press}




\begin{tabular}{|c|l|}
\hline Query No & \multicolumn{1}{|c|}{ Nature of Query } \\
\hline Q13 & Please provide expansion for "TOF SIMS", "MEMS". \\
\hline Q14 & $\begin{array}{l}\text { References Abrams and McBain (1944), Evans et al. (2012), Giannuzzi et al. (2015) are listed in the Ref. list but not } \\
\text { cited in the text, please cite them in the text or delete from Ref. list. }\end{array}$ \\
\hline Q15 & Please confirm whether the provided page numbers in Chee et al. (2014) is correct. \\
\hline Q16 & $\begin{array}{l}\text { Please provide title and retrieved 'date/month and year' in Refs. Dens Solutions B.V. (2016), Hummingbird } \\
\text { Scientific (2016), Protochips Inc (2016). }\end{array}$ \\
\hline Q17 & Please provide retrieved date in the Ref. Kestel (1986). \\
\hline Q18 & Please provide all the co-author names in the Ref. Proetto et al. (2014). \\
\hline Q19 & $\begin{array}{l}\text { More than one reference shares the same author and year-of-publication details. Please check that they have been } \\
\text { correctly differentiated between using a, b, etc. after the year of publication for Ref. Radisic et al. (2006). }\end{array}$ \\
\hline Q20 & Please provide date of the proceedings in the Ref. Young et al. (2008). \\
\hline Q21 & Please provide page range in the Ref. Zhong et al. (2014). \\
\hline
\end{tabular}

\section{Cambridge University Press}




\title{
Sample Preparation Methodologies for In Situ Liquid and Gaseous Cell Analytical Transmission Electron Microscopy of Electropolished Specimens
}

\author{
6 Xiangli L. Zhong, ${ }^{1}$ Sibylle Schilling, ${ }^{1}$ Nestor J. Zaluzec, ${ }^{1,2, *}$ and M. Grace Burke ${ }^{1}$ \\ ${ }^{1}$ School of Materials, Materials Performance Centre. and Electron Microscopy Centre, University of Manchester, Manchester \\ M13 9PL, UK \\ ${ }^{2}$ Argonne National Laboratory, Electron Microscopy Center, Center for Nanoscale Materials, Argonne, IL 60439, USA
}

\begin{abstract}
In recent years, an increasing number of studies utilizing in situ liquid and/or gaseous cell scanning/ transmission electron microscopy (S/TEM) have been reported. Because of the difficulty in the preparation of suitable specimens, these environmental S/TEM studies have been generally limited to studies of nanoscale structured materials such as nanoparticles, nanowires, or sputtered thin films. In this paper, we present two methodologies which have been developed to facilitate the preparation of electron-transparent samples from conventional bulk metals and alloys for in situ liquid/gaseous cell S/TEM experiments. These methods take advantage of combining sequential electrochemical jet polishing followed by focused ion beam extraction techniques to create large electron-transparent areas for site-specific observation. As an example, we illustrate the application of this methodology for the preparation of in situ specimens from a cold-rolled Type 304 austenitic stainless steel sample, which was subsequently examined in both $1 \mathrm{~atm}$ of air as well as fully immersed in a $\mathrm{H}_{2} \mathrm{O}$ environment in the S/TEM followed by hyperspectral imaging. These preparation techniques can be successfully applied as a general procedure for a wide range of metals and alloys, and are suitable for a variety of in situ analytical S/TEM studies in both aqueous and gaseous environments.
\end{abstract}

Key words: in situ S/TEM, liquid cell, gaseous cell, sample preparation, metals, stainless steel, FIB, electropolishing, XEDS, analytical electron microscopy, AEM

\section{INTRODUCTION}

Understanding dynamic processes at nanometer scales during the reaction of materials in liquid and/or gaseous environments can provide opportunities for improved material design in numerous applications. In situ liquid/gas cell scanning/transmission electron microscopy (S/TEM) is a rapidly developing methodology which can provide real-time dynamic micro-to-nanoscale information to study the evolution of a structure, phase distributions, morphology, composition, crystal structure, deformation, corrosion, as well as elemental and interfacial segregation, during the reaction of a material with a controlled environment (Radisic et al., 2006a, 2006b; Zheng et al., 2009; Evans et al., 2011; Chee et al., 2014; Parent et al., 2014; Prestat et al., 2015). Although the concept of a windowed environmental cell (E-Cell) for studying specimens in a gaseous or liquid environment using electron-optical instruments has been recognized for some time, it is only relatively recent that the research and publications related to in situ liquid/gas cell S/TEM studies have begun to grow exponentially (Parsons, 1974; Williamson et al., 2003; Radisic et al., 2006a, 2006b; de Jonge et al., 2009; Proetto et al., 2014; Zaluzec et al., 2014a; Schilling et al., 2015). Since the first report of the development of a sealed fluid cell and its use in a real-time

Received May 20, 2016; accepted September 28, 2016

*Corresponding author. zaluzec@microscopy.com
TEM study of copper precipitation in liquid (Williamson et al., 2003), commercially available liquid and gaseous cells have accelerated research activities involving in situ S/TEM studies. Today, these in situ environmental holders are fabricated by juxtapositioning a pair of environmental chips (E-Chips) having electron-transparent amorphous $\operatorname{SiN}_{x}$ membrane windows supported along their border by a thicker silicon frame. These "E-Chips" are carefully sealed along their periphery to contain the desired media within a small volume which then becomes safely isolated from the vacuum of a conventional electron-optical column. When the path length in the in situ environment is suitably small, electrons can readily pass through both of the $\mathrm{SiN}_{x}$ windows as well as the ensuing media which spans a meticulously engineered gap established between the windows and in which the specimen of interest is immersed. Using advanced MEMS fabrication technologies these E-Chips and their associated holders have also been modified to include electrical connections to facilitate heating, electrochemistry, photoactivation, and modern analytical spectroscopies (Radisic et al., 2009; Zaluzec et al., 2014a; Dens Solutions B.V., 2016; Hummingbird Scientific, 2016; Protochips Inc., 2016). More recently, an alternative type of liquid cell based on entrapment of a very thin liquid film between layers of graphene has also been introduced (Yuk et al., 2012) and facilitates high-resolution imaging, but presently lacks the ability to support flowing/pressurized media. 
The majority of liquid/gas cell S/TEM investigations to date have concentrated upon imaging studies of nanoparticles, nanowires, or sputtered thin films, because of the difficulty in preparing suitable specimens of "bulk" materials, particularly from heterogeneous macroscopic and/ or conventional metallic alloys. The former materials and their studies are of great interest to major fractions of the industrial and engineering community, and as such there is a growing demand to perform in situ TEM examinations in these systems in order to interrogate and understand their real-time behavior and microstructural evolution. The main barrier to in situ E-Cell studies of conventional/bulk metals and alloys has been the difficulty in extracting and placing a suitable macroscopic TEM specimen over the fragile silicon nitride window with the necessary sub-micrometer precision while also forming a hermetic environmental seal in the E-Chips. An added challenge is that the overall thickness of material allowed for insertion between the E-Chips must generally be tailored to fit within a $50 \mathrm{~nm}$ to $5-\mu \mathrm{m}$ gap which delimits the typical media space (i.e., engineered gap) between the $\mathrm{SiN}_{x}$ windows. This is extremely restrictive and challenging requiring precise, delicate sample placement as well as careful thickness control.

S/TEM specimen preparation using focused ion beam (FIB) technology has become increasingly popular and is widely used due to its versatile capabilities, in particular, the ability that it provides to extract site-specific electron-transparent lamellar-shaped specimens having a relatively thin, flat, and uniform thickness profile. Although the ex situ lift-out of the FIB-prepared S/TEM lamella has been used in numerous S/TEM investigations, including some in situ heating and electrochemical property studies (Unocic et al., 2011, 2014), the potential for surface modification and $\mathrm{Ga}^{+}$ion implantation has been raised as a concern (Dalili et al., 2014). It has also been noted that Ga ions can be implanted and mixed into the surface layers of the lamellae (Giannuzzi \& Stevie, 1999; Giannuzzi et al., 2005), and in addition redeposition during FIB sample preparation can modify the structure and composition of the material surface (Matteson et al., 2002). Low-energy argon ion milling after FIB specimen preparation is frequently used to mitigate implantation artifacts for high-resolution electron microscopy (Huang, 2004), however, doing so for the preparation of specimens for in situ E-Cell studies has yet to have been successfully reported. Because of these complications, as well as the absence of a practical procedural approach, the exclusive use of FIB for preparation of the very thin lamella required for in situ environmental studies has seen limited application. In contrast, conventional twin-jet electropolishing of metallic specimens is a well-understood and controlled process and, when carried out properly, can mitigate FIB-related surface modification and $\mathrm{Ga}^{+}$ion implantation issues. Unfortunately, preparation of uniformly thin and small lamella is not the strength of this preparation methodology. Herein, we report on the development of specimen preparation methods that combine both electropolishing and FIB. This methodology incorporates the advantages of the two disparate techniques and has been used to produce site-specific samples with large electron-transparent areas suitable for both gaseous and a fully hydrated environmental studies in E-Cell systems operating at pressures up to $1 \mathrm{~atm}$ (Zhong et al., 2014).

\section{Materials and Methods}

Electron-transparent specimens for this work were prepared using a combination of conventional electrochemical jet-polishing and FIB extraction techniques, both of which are described below in detail. In this work, a Struers TenuPol-5 twin-jet electropolishing unit and a Jubalo closed cycle refrigeration system were used for conventional thin-foil electrochemical preparation, whereas site-specific sample extraction was performed using an FEI Quanta 3D Dual-Beam FIB/SEM. Equivalent results should be reproducible with similar equipment from alternate manufacturers. Microstructural and hyperspectral imaging of the resulting electron-transparent specimens were conducted in an FEI Tecnai T20 Analytical Electron Microscope (AEM) operated at $200 \mathrm{kV}$ and equipped with an Oxford Instruments X-max 80 TLE windowless Si drift detector and an AZTEC analyzer for conducting hyperspectral imaging using X-ray energy-dispersive spectroscopy (XEDS). The in situ holder used in this study was a Protochips Poseidon P210 liquid E-Cell using ambient air and deionized $\mathrm{H}_{2} \mathrm{O}$ as the environmental media (Zaluzec et al., 2014a). The E-Cell windowed E-Chips (300- $\mu$ m-thick Si frame, having $\sim 50$-nm-thick $\mathrm{SiN}_{x}$ windows covering rectangular openings of up to $500 \times 50 \mu \mathrm{m}$ ) were commercially procured from Protochips Inc. Before use, these E-Chips were cleaned according to the manufacturer's recommendations and in addition, a Hitachi ZONE-SEM was used to remove any residual hydrocarbon contamination from the E-Chip surface before attaching the electron-transparent specimens of interest. This last step also made the E-Chip $\mathrm{SiN}_{x}$ surface hydrophilic. We note that any low power plasma cleaning system can also be employed to remove residual hydrocarbon layers without damaging the $\mathrm{SiN}_{x}$ windows.

\section{Specimen Preparation Methods}

The methodology developed in this study consists of four basic steps:

1. Preparation of conventional electropolished TEM thin-foil specimens.

2. Identification and FIB cut out of the region of interest (ROI) from the electropolished specimens.

3. Attachment of the FIB plan-view sections to the E-Cell windows:

a. Pt deposition-attachment "Cut-and-Attach" method; and

b. Adhesive-attachment "Cut-and-Adhere" method.

4. Sealing and mounting of the E-Cell in the in situ specimen holder.

Details for each of these procedures will be presented next. 
204

\section{Electrochemical Twin-Jet Polishing}

Cold-rolled Type 304 stainless steel (SS) was used as the test material in this work. Slices $\sim 0.7 \mathrm{~mm}$ in thickness were cut from the Type 304 bulk steel using a Struers Accutom-5 sectioning system. These slices were next mechanically thinned (using SiC grinding papers) to $\sim 100 \mu \mathrm{m}$ in thickness before punching 3-mm-diameter disc samples. Conventional TEM specimens were obtained by electropolishing the 304 SS in 20 vol\% $\mathrm{HClO}_{4}-80 \% \mathrm{CH}_{3} \mathrm{OH}$ at $-33^{\circ} \mathrm{C}$ and $20 \mathrm{~V}$, with automated cut-off upon reaching electron transparency as detected using the automatic sensor of the polishing unit. Although this experimental procedure is specific to preparation of the Type 304 SS material used as part of this methodology, corresponding electropolishing procedures for many metallic materials can be substituted (Kestel, 1986).

\section{Identification of ROI and "Plan-View" FIB Extraction}

After electropolishing to TEM quality, the thin-foil SS specimens were transferred onto FIB/SEM/STEM imaging compatible sample holders for the FEI Quanta 3D Dual-Beam FIB/SEM used in this work. The location of the electron-transparent area of interest for in situ TEM analysis could be readily determined using moderate energy (20-30 kV) STEM imaging in the FIB/SEM. Typical electron beam bright-field images of such a thin area of interest, which can be acquired at the coincident point of the electron and ion beam, are illustrated in Figures $1 \mathrm{a}$ and $1 \mathrm{~b}$. In the absence of an STEM detector system in an FIB/SEM, secondary electron imaging can also be used for identifying the electron-transparent area (Young et al., 2008) as these regions will appear significantly brighter than the thick (non-electron transparent) regions (Fig. 2a). This increased signal results from the fact that at $20-30 \mathrm{kV}$ the electrontransparent areas of "thin" TEM specimens have a second source for secondary electrons, namely those emitted from the exit surface of the thin regions, creating an additional source for emission of secondary electrons, relative to the thicker (non-transparent) area.

After the electron-transparent area of interest is identified, careful eucentric point alignment of the holder and specimen relative to the electron/ion beam optical axis is carried out. To accomplish this step in the selected dual-beam FIB/SEM, the ion beam is blanked and the sample on the STEM specimen holder is first tilted (typically $52^{\circ}$ ) toward the ion
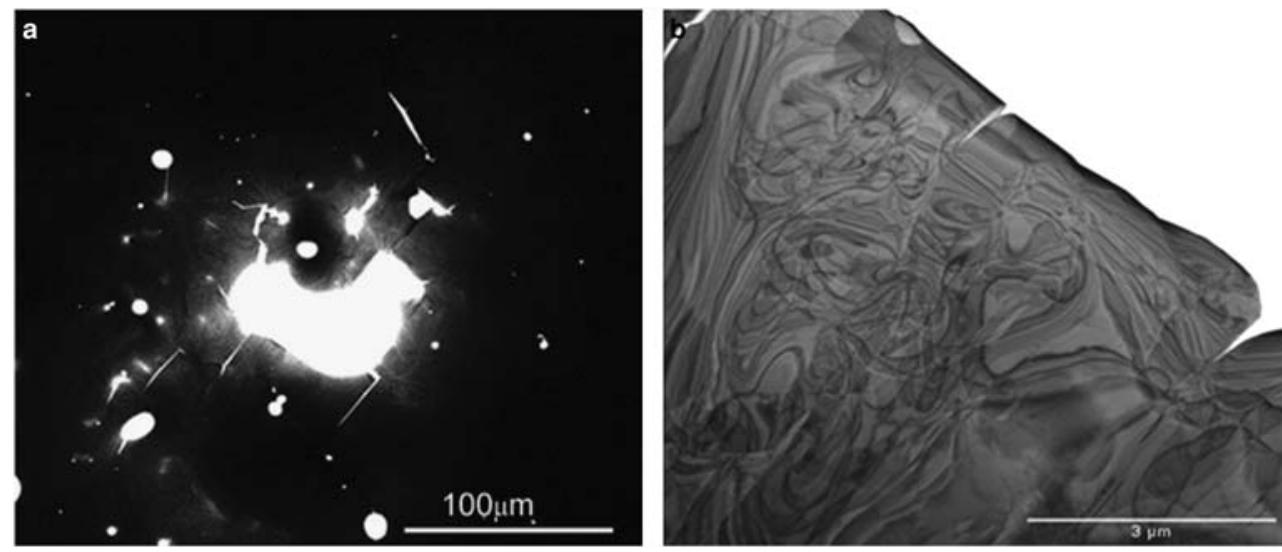

Figure 1. Electron beam $30 \mathrm{keV}$ scanning transmission electron microscopic (S/TEM) bright-field (BF) image shows the thin area of interest: (a) BF image, (b) higher magnification BF STEM image showing preservation of thin electron-transparent area at $30 \mathrm{keV}$.
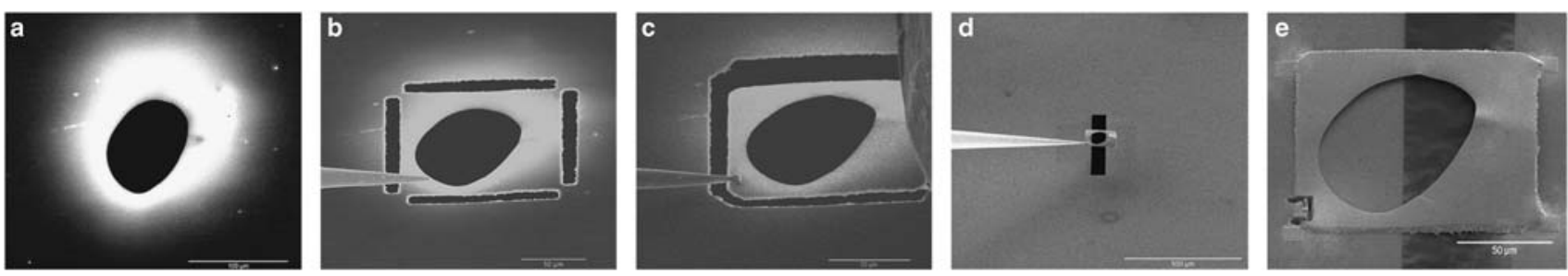

Figure 2. Electron beam images of "Cut-and-Attach" approach: (a) scanning transmission electron microscopic dark-field image showing the electron-transparent region, (b) focused ion beam slots cut around the periphery of the region of interest, with corner attachments still in place. c: Micromanipulator attached to the area of interest and freed by milling off supporting material, (d) sample positioned above the $\mathrm{SiN}_{x}$ window, (e) micromanipulator cut free by milling away the surrounding material after corner attachment to the environmental chips by organometallic $\mathrm{Pt}$ (om-Pt) deposition. The observant reader will notice a diffuse patch of material surrounding the perimeter of the small rectangular tabs affixing the corners of the cut out. This is residual om-Pt discussed in the text. 
beam column axis. Following tilting, fine tuning of the FIB coincident point of the ion beam and electron beam is carried out on a sacrificial area of the specimen. To achieve this, the specimen is next translated a few hundred micrometers away from the ROI, along a direction parallel to the stage tilt axis. This approximately retains the eucentric axis, however, it should be realized that this only holds true for relatively flat unbent specimens. Specimens which are significantly misshapened, bowed, or angled should be meticulously avoided as they pose a risk of breaking the $\mathrm{SiN}_{x}$ isolation windows when later mounted in the E-Cell, equally importantly the location of the electron/ion beam coincidence point in the proceeding step can become compromised. Next, the ion beam is unblanked and a small sacrificial area is milled at low current $(\sim 0.4 \mathrm{nA} /$ $30 \mathrm{kV}$ ) in spot mode for several seconds, then the ion beam reblanked. The FIB/SEM stage is next moved in the $Z$ direction until the milled area is recentered to the electron imaging screen and nominally defines a coincident electron/ion beam point. This step is iterated as necessary until convergence is achieved. At this juncture, the original ROI of the specimen is translated, without height adjustment and with the ion beam blanked, back to the optic axis and the ROI is ready to be defined and extracted. This procedure by design results in minimal exposure of the ROI to the adverse effects of the ion beam and its associated sputtering/damage.

Next, the electron-transparent portion of the electropolished TEM disc containing the ROI is delimited (cut out) by site-specific $\mathrm{Ga}$ ion beam milling (Fig. 2b) using the microprocessor-controlled ion beam blanking and scanning system of the FIB/SEM. The specific cutting procedure employed at this juncture is carefully orchestrated as the milling process and plays a key role in the overall success rate of sample preparation. The goal here is to use site-specific milling to cut out a specific ROI from the electropolished specimen and to then use FIB lift-out techniques to transfer this to an $\mathrm{SiN}_{x}$ window. Ultimately, the area of interest will be carefully relocated and attached to the interior surface of either the electron entrance (top) $\mathrm{SiN}_{x}$ window for STEM/ XEDS studies, or on the electron exit surface (bottom) window for TEM/electron energy-loss spectroscopic (EELS) studies of the respective E-Chips used in the in situ holder. The selection of mounting on the entrance or exit window is determined by the experimental measurements planned and should be chosen to mitigate the effects of scattering upon the respective imaging and spectroscopy modes employed.

Avoiding/minimizing Ga deposition/contamination of the TEM specimen during the cut out and extraction step by using reduced ion current during FIB operations on the electropolished TEM sample is a critical aspect of this procedure. It is important to recognize that it is virtually impossible to not implant Ga in a TEM specimen whenever an area is illuminated by the focused Ga ion beam. Thus, to the greatest extent possible the critical areas of the specimen to be studied in the E-Cell should be viewed principally by electron beam imaging. With this in mind, it should be realized that it will be unavoidable that during the FIB 282 cut-out process that for some fraction of time key ROI may be exposed to the ion beam, this needs to be minimized to the greatest extent possible. Setting up a workflow that achieves this is a challenge and necessarily will have to be customized to the instrument and operator expertise.

The following few salient points are appropriate for implementing such a workflow. First, the identification and delimitation of the milling path should be done under electron beam observation. Recall that in the preceding step the coincidence alignment of the electron/ion beam was done on a sacrificial area far removed from the ROI with this specific purpose in mind. Second, in order to minimize Ga implantation/contamination of the pristine electrontransparent, electropolished area, the perimeter of the cut-out edge should be intentionally located as far away as is practical from the critical ROI. These criteria, together with the dimensions of the $\mathrm{SiN}_{x}$ window whose dimensions depend upon the application and manufacturer (ranging from $20-50-\mu \mathrm{m}$ wide to $200-500-\mu \mathrm{m}$ long), become key controlling factors in an operator's decision of the areal dimensions of the cut-out region. Third, it is recommended that one targets to cut out a section having at least one supporting dimension twice as wide as the minimum dimension of the $\mathrm{SiN}_{x}$ window. Thus, for a $50-\mu \mathrm{m}$-wide $\mathrm{SiN}_{x}$ slot window, a typical section would nominally be $\sim 100-\mu \mathrm{m}$ wide, whereas the perpendicular dimension should be of at least a similar dimension while consistent with the ability of the FIB to mill the specimen within an appropriate time frame. Fourth, at the nominal center of the cut out should be the ROI to the greatest extent possible. Oversizing the cut out and creating a moderately large and thick electron-opaque zone surrounding the thin ROI is advantageous as it leaves sufficient material on the perimeter for handling and attachment to the Si frame of the E-Chip, as will be illustrated shortly. However, the thickness of this perimeter should not be greater than the E-Cell gap, otherwise a hermetic seal of the E-Cell may not be achieved. A larger rectangular cut out also provides ready access to three of its corners which are unobstructed and well removed from the ROI, while the fourth corner becomes available for micromanipulator attachment. Fifth, it is also essential to balance the milling time required for the cut out with the ion current employed to optimize the speed and minimize any inadvertent/potential damage to the large and thin electrontransparent area. An ion beam voltage of $30 \mathrm{kV}$ was used throughout this work, with ion beam milling currents typically being 3-5 nA. Finally, checking the electron-transparent area after ion beam extraction using XEDS for the absence of Ga signal should be a final step before any further work in the E-Cell. If Ga is detected then it is incumbant upon the analyst to assess its contribution to any microstructural evolution studies and/or as appropriate to remake the section.

An example of the results of FIB milling defining a cut-out area and illustrating this idea is shown in Figure 2b. Visible in Figure 2b are the uncut corners used to support the electron-transparent region, while the dimensions of the rectangular area $(\sim 150 \times 75 \mu \mathrm{m})$ provide adequate mechanical robustness and stability for micromanipulator attachment and eventual fastening to the E-Chip Si frame. FIB lift-out and 
attachment to the E-Chip is the next step in the workflow and two different procedures are summarized next.

\section{FIB Lift-Out: Pt Deposition-Attachment: “Cut-and- Attach" Method}

In the Cut-and-Attach method, FIB lift-out is accomplished using organometallic $\mathrm{Pt}(\mathrm{om}-\mathrm{Pt})$ deposition/attachment of a micromanipulator to the ROI and is identical in all important metrics to the procedure used in traditional cross-section FIB lift-out specimen preparation (Mayer et al., 2007), with the small exception of the orientation of the section to be retrieved which is dictated by the attachment process and location on the E-Chip. At this phase of the process, a micromanipulator is inserted into the FIB/SEM chamber and is carefully maneuvered to the one corner/edge of the electron-transparent area that is still connected to the electropolished TEM disc specimen at corner points around the rectangular perimeter (Fig. 2b). Skilled FIB operators should be able to approach the sample using only electron imaging without using ion beam imaging, assuming that the previously outlined procedure of local coincident point alignment has been carried out carefully, the major difficulty being associated with the orientation of the cut out relative to the manipulator motion. During this step, adjustment of the electron beam current and contrast/ brightness can help enhance the shadow of the probe which can be used to assist approaching the sample. Once the tip is successfully touching a corner of the section, localized om-Pt deposition is used to affix the corner of the cut-out area to the tip of the micromanipulator (Fig. 2c). To accomplish this, we have successfully employed both a $0.05-0.3 \mathrm{nA} \mathrm{Ga}$ ion beam at $30 \mathrm{kV}$ or $3-5 \mathrm{nA}$ electron beam at $5 \mathrm{kV}$ to perform the $\mathrm{om}-\mathrm{Pt}$ deposition. Utilizing the smallest beam diameter with a minimum beam tail is a noteworthy consideration at this step. If a large beam tail (or beam skirt) is present then some (residual) measure of nonlocalized om-Pt deposition can occur outside of the desired deposition location (see, e.g., Fig. 2e). This residual om-Pt (which also contains significant hydrocarbon) can become a source of both $\mathrm{Pt}$ and $\mathrm{C}$ contamination at a later stage of any in situ experiments and thus it's minimization is not without merit. In addition, we note that the om-Pt vapor which is literally sprayed out of the gas injection system needle during the deposition step will by its very nature also become dispersed over a relatively large area (hundreds of square micrometers) in the vicinity of the needle-shaped gas jet. The presence of this flowing gaseous om-Pt vapor over the specimen cannot be avoided, and it is not without concern that this vapor can traverse large distances and possibly be absorbed in the immediate surface of the previously pristine electropolished specimen. To minimize the adherence of surface-absorbed $o m-\mathrm{Pt}$ on the specimen, electron beam imaging of the ROI immediately after om-Pt deposition following deposition should be avoided to the greatest extent possible to allow mobile species to be carried away by the pumping system. The experienced FIB/SEM operator will be the best judge of the most effective procedure for such minimization in their respective instrument, however, in the absence of a specific cleaning protocol, waiting for full vacuum recovery after the om-Pt vapor source is isolated, is a reasonable procedure before renewed electron imaging, and is the technique used herein. Once the micromanipulator is attached to the ROI section, the remaining three supporting tabs at the corners of the thick opaque areas can be readily FIB cut free from the electropolished foil as is illustrated in Figure 2c. Normal sample extraction and micromanipulator relocation is ready to be conducted (Figs. $2 \mathrm{~d}$ and 2e).

\section{FIB Lift-Out: Adhesive-Attachment: "Cut-and- Adhere" Method}

As an alternative to the om-Pt attachment step outlined above, we have also successfully made use of the adhesion force of an epoxy-coated micromanipulator (OmniProbe; Oxford Instrument) to remove the delineated ROI. This Cut-and-Adhere procedure was developed to mitigate potential issues associated with the deposition of om-Pt-rich compound in the vicinity of the specimen as discussed in FIB Lift-Out: Pt Deposition-Attachment: "Cut-and-Attach" Method section. In this approach, a thin epoxy coating is intentionally applied to the micromanipulator's extraction tip in order to increase the adhesion force of the tip by slightly coating the tip. Before coating, the surface of the micromanipulator tip is first irradiated with the ion beam at $\sim 30 \mathrm{kV} / 15 \mathrm{nA}$ for $2 \mathrm{~min}$ to clean (remove surface oxide) and also to slightly "roughen" its surface. We have found that a suitable coating can be made using commercial superglue (Loctite; Henkel, UK), and can be applied by simply dip-coating the micromanipulator. This coating is applied externally from the FIB/SEM vacuum chamber and is typically allowed to dry for $\sim 2 \mathrm{~h}$, after which the coated needle is simply reinserted into the micromanipulator system. Using the coated and now mildly insulating micromanipulator tip specimens can be "picked up" from an area and relocated onto the E-Chip window without employing om-Pt deposition/attachment. It is not clear if this adherence of the tip to the extracted foil is related to the presence of electrostatic and van der Waals forces or if it is due to the fact that the epoxy is still mildly "sticky," the mechanism by which this occurs is not important at this point in time. After lift-out and transferral to the $\operatorname{SiN}_{x}$ window, the specimen must still, however, be anchored to the $\mathrm{Si}$ frame by means of om-Pt deposition to avoid sample movement/drift during in situ TEM observation in any media. The principle of minimalist om-Pt deposition as described earlier should again be applied here. Fundamentally, the use of minimal electron beam or low ion beam current om-Pt deposition should be used throughout the procedure in order to decrease the potential for $\mathrm{om}-\mathrm{Pt}$ contamination.

An alternative approach to coating the micromanipulator tip with adhesive external to the FIB/SEM is to use an electron beam hardenable glue as the micromanipulator's adhesive which can be utilized within the FIB/SEM instrument. Similar in principle to the preceding method, the purpose is also to 
minimize the use of om-Pt for attachment of the cut-out ROI to the $\mathrm{SiN}_{x}$ window. As previously, the specimen area of interest is identified using electron imaging (Fig. 3a). Next, the micromanipulator tip is touched onto a vacuum compatible glue which is co-located within the specimen chamber (SEM glue from Kleindiek Nanotechnik). The advantage here is that the process can be readily observed in real time under a low current $(<0.1 \mathrm{nA})$ electron beam (Fig. 3b). The micromanipulator tip which is now lightly endowed with a thin layer of "glue" is next maneuvered to the partially cut-out ROI, and the glue-rich tip is allowed to touch an isolated corner of the ROI section. It is then affixed by electron beam hardening under a higher current $(0.5-1 \mathrm{nA})$ probe. The cutout sample, which is now attached to the micromanipulator needle by the hardened glue, can next be cut free by FIB milling and subsequently lifted out (Fig. 3c). To affix the lift-out section to the $\mathrm{SiN}_{x}$ window, a corner of the cut-free sample edge can also be manipulated so as to gently brush into the SEM glue while still within the FIB chamber (Fig. 3d). As previously described, the sample can be next relocated to the E-Chip window using the micromanipulator and finally held in place by contacting the glue-rich corner to the bulk silicon support area of the E-Chip (Fig. 3e) and beam hardened, all of which must be done while judiciously avoiding the fragile $\mathrm{SiN}_{x}$ window. The advantage of this hardened glue method is the minimization of om-Pt usage during the entire specimen preparation process. The disadvantage of using hardened glues is the great care needed to touch the extracted ROI to the SEM glue without damaging/bending the very fragile cut out.

\section{Mounting the FIB Lift-Out to an $\mathrm{SiN}_{\boldsymbol{x}}$ E-Chip}

To accommodate the preceding steps of relocating the cut out to the E-Chip window described above, it was necessary to fabricate a custom E-Chip holder. This is readily accomplished by modifying a standard pin-type SEM stub and enables holding and the subsequent mounting of the electropolished section onto the $\mathrm{SiN}_{x}$ window E-Chip. Figure 4 shows the photograph of our modified SEM stub (termed the E-Chip holder) used for attaching the electron-transparent specimen to the E-Chip. Before transfer of the cut-out sections of the electropolished thin films, the appropriately selected E-Chip is slid into the customized/grooved holder. When mounting this holder into the dual-beam FIB its pairs of parallel grooves into which the E-Chips are located are oriented parallel to the FIB/ SEM tilt axis so that the chip will be mechanically stable during any tilting operations. This can be avoided if the E-Chips are affixed to the stub by an appropriate procedure (glue, tape, clamps, etc.). However, it was found that simple care in handling is more than sufficient. For the Poseidon system used in this work, two different sizes of silicon frames are employed in the in situ holder design, a large upper chip (green in Fig. 4) and a smaller lower chip (yellow in Fig. 4); the set of grooves in this E-Chip holder are of dimensions needed to accommodate both sizes of E-Chip. Upon close inspection of Figure 4, the reader will be able to discern the long $\mathrm{SiN}_{x}$ slot window (gray) on the chips, and in particular should notice the orientation of this slot with respect to the holder tilt axis and the slot (red arrow).

The extracted ROI, which has been attached to the manipulator tip by either om-Pt deposition or adhesion, is then transferred to the selected (top or bottom) E-Chip that is loaded onto the aforementioned E-Chip holder. Here, the selected E-Chip of the in situ cell pair is first slid into the grooved E-Chip holder, with the groove aligned parallel to the tilting axis of the dual-beam FIB stage as indicated in Figure 4. Next, the cut-out ROI, which is attached to the manipulator tip, is carefully relocated/positioned over the appropriate $\mathrm{SiN}_{x}$ window, and is aligned carefully over the nominal $50-\mu \mathrm{m}$-wide slotted window. Recalling that this cut out has an intentionally larger width $(\sim 100 \mu \mathrm{m})$, its terminal edges extend beyond the complete $\mathrm{SiN}_{x}$ window onto the $\mathrm{Si}$ support frame (Fig. 2d). During the final om-Pt attachment processes of the cut out to the $\mathrm{Si}$ frame, the large overhang minimizes any possible contamination near the ROI and also avoids inadvertent milling and damaging of the extremely thin and fragile $\mathrm{SiN}_{x}$ window. Once in position, a small rectangular area $(\sim 3 \times 5 \mu \mathrm{m})$ of low ion beam om-Pt deposition or adhesion is used to attach the corners of the "cut out" to the Si support base (om-Pt illustrated in Fig. $2 \mathrm{e}$ and adhesion in Fig. 3e). Finally, after the specimen is secured the micromanipulator needle tip is cut free from the sample using FIB.

\section{E-Cell Assembly and Demonstration}

To demonstrate the efficacy of this methodology to mount and observe electropolished TEM specimens in both a
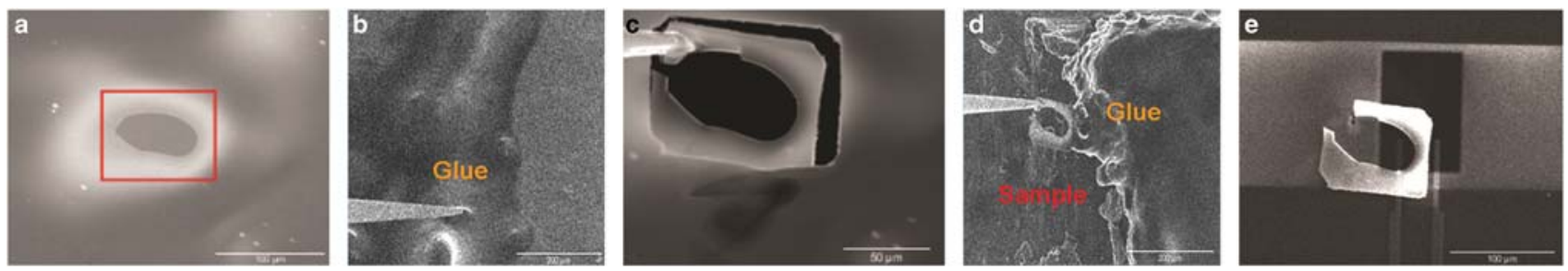

Figure 3. "Cut-and-Glue" method: (a) electron beam secondary electron image of the electron-transparent area in the electropolished transmission electron microscopic foil, (b) manipulator positioned to pick up the glue deposit, (c) electron beam-hardened glue attached the sample to the manipulator tip and sample was cut free from the electropolished disc, (d) cut-free sample edge brushed/touched into the glue deposit to pick up a small amount, and (e) released sample affixed using glue onto the $\mathrm{SiN}_{x}$ window. Note the two vertical structures visible on either sides of the glued cut out are electrical feedthroughs for use as part of electrochemical cell experiments. 


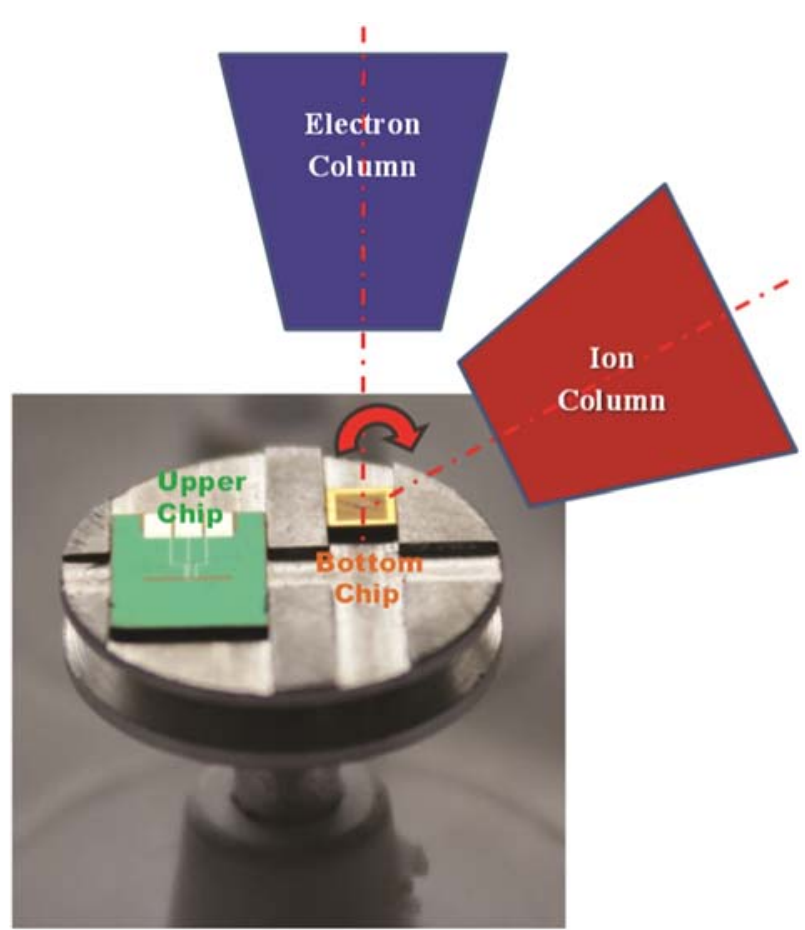

Figure 4. Photograph of the modified environmental chip (E-Chip) holder used for attaching the electron-transparent specimen to the E-Chip. The selected E-Chip is slid into the appropriate-sized groove on the holder. The dual-beam focused ion beam tilting axis is aligned to be parallel to grooves of the holder. For the Poseidon system used the E-Chip sizes are different for the upper $(4.5 \times 6 \mathrm{~mm})$ and lower $(2 \times 2 \mathrm{~mm})$ chips.

gaseous as well as a flowing liquid, we have selected an alloy of interest, Type 304 austenitic SS, which is generally resistant to corrosion but will oxidize in appropriate water environments (Ziemniak \& Hanson, 2002), and for which a detailed in situ study will be reported in a follow-up publication. After a conventional TEM specimen was first prepared by twin-jet electropolishing, and the electrontransparent region was "cut out" using the FIB, the extracted sample was carefully attached to the bottom E-Chip surface. Care was taken to insure that the maximum thickness of the FIB section selected was $<500 \mathrm{~nm}$, and was judged by simple end-on SEM observation in the FIB/SEM. As discussed earlier, since TEM was the primary imaging modality of this example, attachment to the bottom E-Chip was selected for the mounting step. For the in situ holder system used in this study, an integrated prefabricated spacer on the lower chip introduces a 500-nm gap between the windows (Fig. 5). The selected 500-nm gap is sufficient to hold both the cut-out ROI as well as either ambient air or liquid $\mathrm{H}_{2} \mathrm{O}$ without rupturing the window of the E-Cell under $1 \mathrm{~atm}$ of pressure. Although narrower E-Cell gaps are both available and advantageous from the viewpoint of imaging and spectroscopy, they also create more difficult preparation conditions, as the total thickness of the extracted electron-transparent specimen plus any "attachment" material (i.e., om-Pt or glue) must not be so thick as to inadvertently rupture either of the

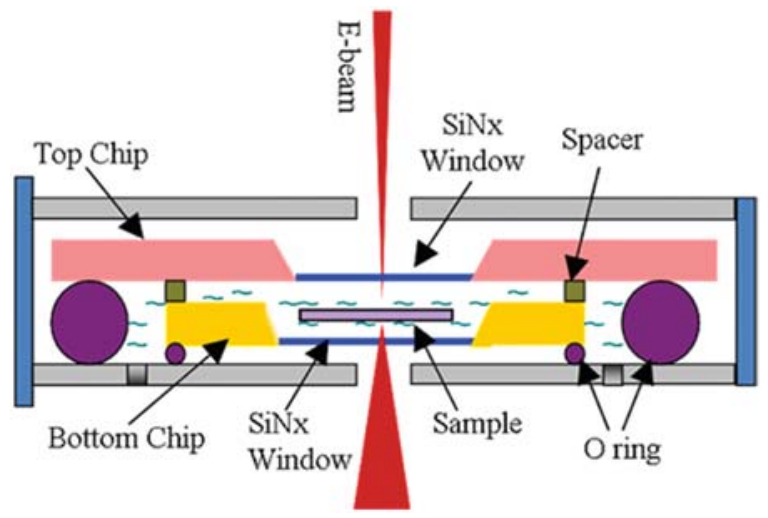

Figure 5. Schematic drawing of the P210 liquid cell (Protochips Inc.) in which the sample is placed in-between the top and bottom chips that are separated by an engineered spacer creating a nominal 500-nm gap, and isolated from the transmission electron microscopic vacuum environments by means of a pair of small and large $\mathrm{O}$ rings.

$\mathrm{SiN}_{x}$ windows during assembly. We also note that because the thin-foil section is attached to the $\mathrm{Si}$ support, a small space between the specimen and the lower $\operatorname{SiN}_{x}$ window nearly always exists for these methods, thereby allowing either gas or liquid to flow across both sides of the electropolished specimen, this is particularly important to insure full gas/liquid/solid reactions occur uniformly. Positioning of the specimen along the length of the slotted window is done at the operator's discretion. Depending upon the experiment being conducted and the amount of tilting required for possible spectroscopy, it can be appropriate to offset the section from being centered on the long slot to mitigate XEDS detector shadowing effects.

\section{Example TEM and AEM Observation}

Having successfully described a systematic set of procedures for sectioning, transporting, assembling, and sealing an electropolished TEM specimen into an in situ E-Cell, in this last section we end by demonstrating the methodology as part of the initial stages of a study of localized dissolution in Type 304 SS in both air and in pure water. In Figures 6a and $6 \mathrm{~b}$, we first present a series of images of the single-tilt in situ holder loaded with a cut-out specimen in an $\operatorname{SiN}_{x}$ E-Cell as examined in the TEM with $1 \mathrm{~atm}$ of air surrounding the specimen prepared using the Cut-and-Attach method as detailed above. The atmospheric pressure surrounding the specimen was simply achieved by opening the tubing normally dedicated to transporting liquid to ambient air and allowing equilibration to occur. This was done to not only verify the integrity of the cell to hold pressure, but also to facilitate preliminary TEM observations with minimum multiple scattering from the media surrounding the specimen. Conventional diffraction contrast experiments can be performed in this mode although they are restricted in nature for two reasons: (1) the in situ E-Chip holder itself is a 

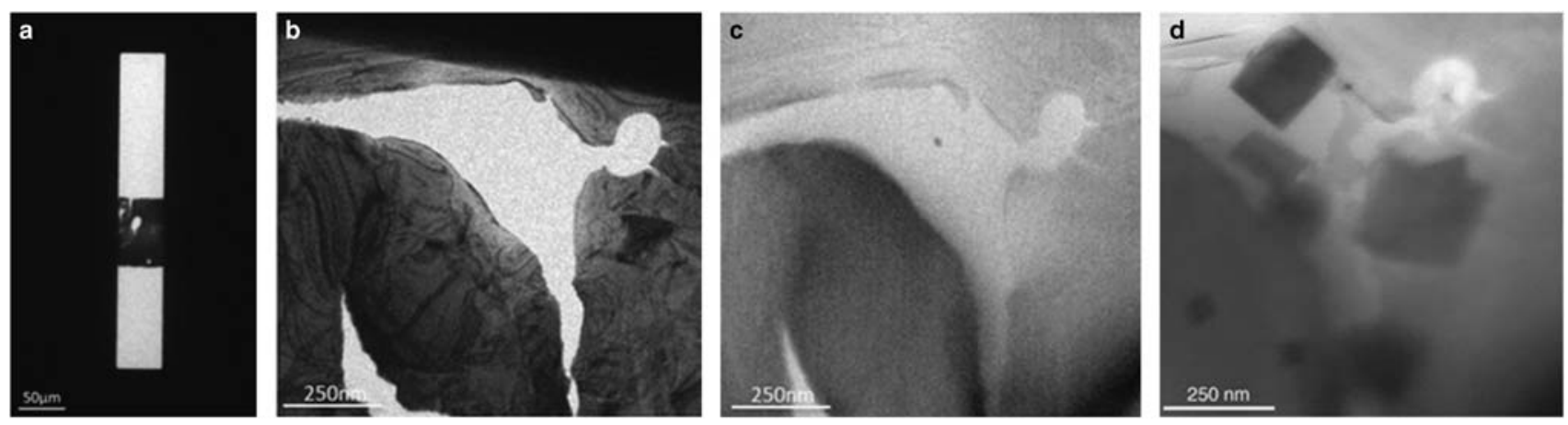

Figure 6. a: Low-magnification bright-field transmission electron microscopic (TEM) image of hybrid sample and liquid cell window, (b) TEM image in 1 atm air, (c) TEM image of steel sample immersed in $\mathrm{H}_{2} \mathrm{O}$, (d) microstructural changes to (c) occurring after $27 \mathrm{~h}$ of exposure to room temperature $\mathrm{H}_{2} \mathrm{O}$.

single-tilt device, and (2) measurements are limited because of the multiple scattering in the $\mathrm{SiN}_{x}$ windows as well as the presence of $\sim 500 \mathrm{~nm}$ of $1 \mathrm{~atm}$ of air, both of which affect the image quality. Figure $6 \mathrm{c}$ illustrates the effects of the introduction of $\mathrm{H}_{2} \mathrm{O}$ carefully into the cell, which gradually displaces the air present. The presence of liquid can be recognized by comparing Figure $6 \mathrm{~b}$ (air) versus Figure $6 \mathrm{c}$ (water). The reduced contrast and blurring is a direct result of multiple scattering of the electron beam in the liquid media both above and below the thin TEM section. It should also be immediately apparent that the liquid media virtually obscures any electron diffraction measurements during in situ liquid studies. This could, of course, be partially compensated for by either increasing the electron beam energy and/or decreasing the $\mathrm{SiN}_{x}$ window and liquid media thickness. After initial confirmation of the integrity of the E-Cell, the specimen was allowed to remain in flowing water (with the electron beam blanked) for $\sim 27 \mathrm{~h}$ at room temperature, this was done to assess any liquid corrosion/ oxidation without the complicating influence of electron beam effects. Figure 6d shows the microstructural changes that ensued after the extended exposure to liquid water: specifically, one observes the formation of discrete "blocky" structures, which, in this example, appeared as rectilinear precipitates on the sample after prolonged exposure in water, as well as bending/distortions of the thin metal film. As the specimen was mounted on the lower window in anticipation of TEM imaging experiments, this configuration is not optimum for STEM/XEDS, and unfortunately once the specimen configuration is completed it cannot be disassembled and reconfigured on the upper window which is best for X-ray spectroscopy. Nevertheless, although less than optimum, in situ XEDS measurements in the liquid cell can be conducted and provide a qualitative identification of these coarse discrete microstructural features as $\mathrm{Fe}-\mathrm{Ni}$-enriched Cr-poor oxides (Schilling et al., 2014). The presence of liquid water is additionally verified by the observation of small items of occasional floating debris, but more readily by measuring the magnitude of the $\mathrm{SiK} \alpha / \mathrm{OK} \alpha \mathrm{X}$-ray line ratio whose magnitude varies as the media changes from vacuum, to air, to water (Zaluzec et al., 2014b). We also note that elemental core-loss imaging using EELS is not feasible during our liquid water experiments as the relative mean free path $t / \lambda$ under these conditions is significantly $>4$ and at these values of thickness (i.e., $t / \lambda>>1$ ) core-loss imaging by EELS becomes impractical (Holtz et al., 2013; Zaluzec et al., 2014b). Fortunately, X-ray hyperspectral imaging (Fig. 7) is not as adversely affected by the presence of liquid water as is EELS and the distributions of the elemental species is readily visualized by simply integrating the full-width at half-maximum of the $\mathrm{O}, \mathrm{Fe}, \mathrm{Ni}$, and $\mathrm{Cr}-\mathrm{K} \alpha$ XEDS peaks as a function of STEM probe position. Their measurement confirms that the rectilinear precipitates were enriched in $\mathrm{O}$, $\mathrm{Fe}$, and $\mathrm{Ni}$ and depleted in $\mathrm{Cr}$. The nature of the precise oxide formed could not be established using electron diffraction due to the amount of liquid water present, which as stated earlier precludes all diffraction studies and also contributes significantly to the $\mathrm{O}-\mathrm{K}$ signal. As can be observed in the sum spectrum of Figure 7, a small amount of Pt was detectable in our XEDS data, which we attribute to the om-Pt used to attach the specimen onto the $\mathrm{Si} / \mathrm{SiN}_{x}$ window and is not surprising. Importantly, no Ga was detected in the spectral analyses (Fig. 7, inset), which is also consistent with an optimized and careful FIB milling procedure. Although no Ga signal was detected in the XEDS spectrum, we cannot assume it is completely absent, only that it is less than the detection limit of the XEDS technique in this experimental configuration. Giannuzzi et al. (2005) has found that after FIB processing small amounts of Ga can be present millimeters away using TOF SIMS. Thus, it would not be surprising if small amounts were present but not detected.

\section{Summary And Conclusions}

In summary, a novel set of hybrid electrochemical-FIB sample preparation procedures for the production of S/TEM samples from bulk metals/alloys has been developed to facilitate environmental S/TEM studies. These methodologies have been successfully applied to in situ environmental S/TEM observations of extracted electropolished specimens immersed in both 


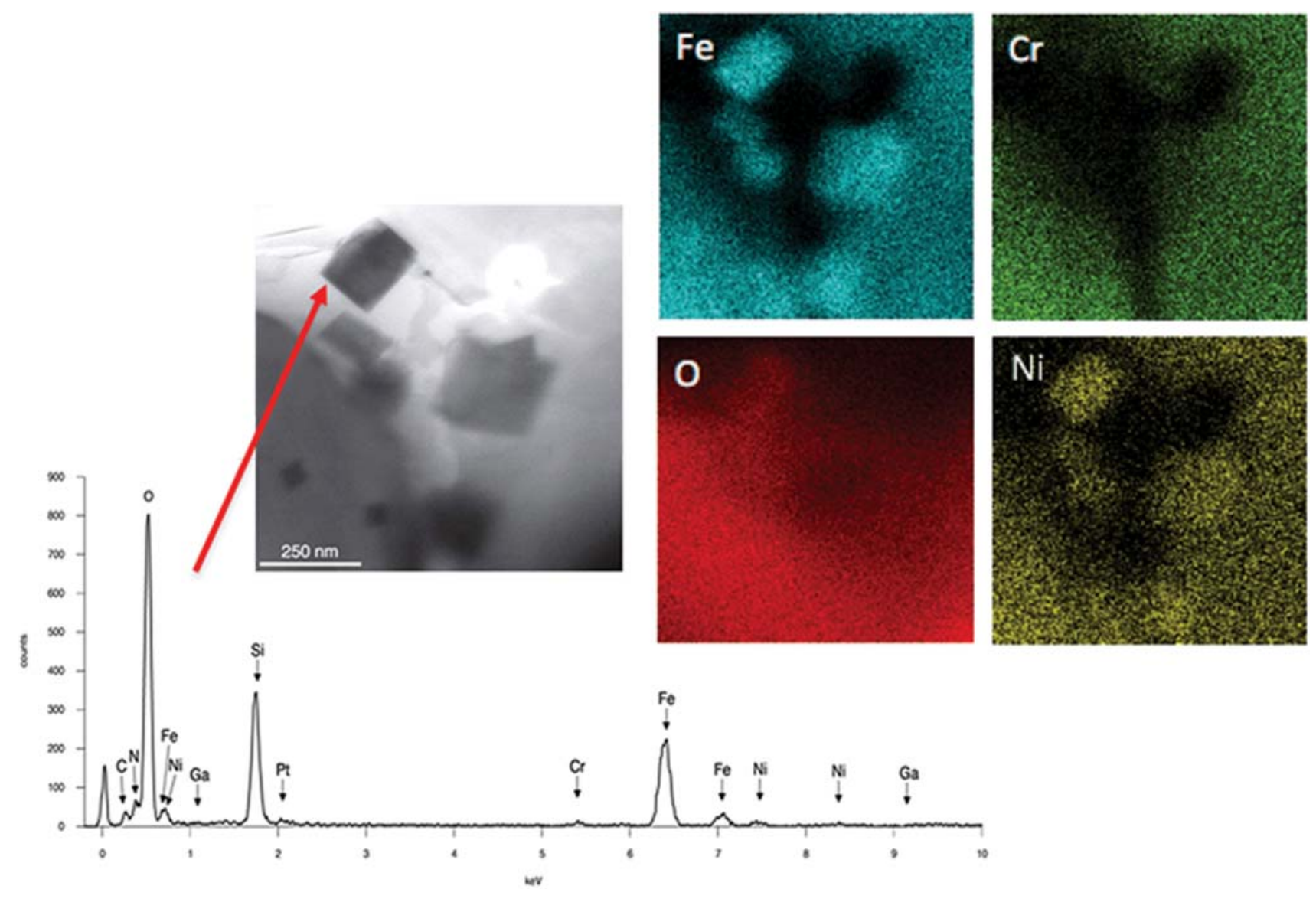

Figure 7. Hyperspectral images obtained from the Type 304 stainless steel specimen in $\mathrm{H}_{2} \mathrm{O}$ showing the presence of FeNi-rich phases (Schilling et al., 2014). Colorized insets are hyperspectral elemental images from $\mathrm{Fe}$ (blue), $\mathrm{Cr}$ (green), $\mathrm{Ni}$ (yellow), and $\mathrm{O}$ (red) $\mathrm{K} \alpha \mathrm{X}$-rays, together with a sum spectra calculated from all pixels. The spectral inset show a sum spectra from the hyperspectral experiment with the characteristic spectral elements identified, no detectable Ga was observed above background levels.

ambient air and in $\mathrm{H}_{2} \mathrm{O}$, and can be used to prepare a wide range of samples for other unique in situ environmental S/ TEM and in situ hyperspectral imaging experiments. The procedures outlined herein provide the community with a reproducible method of preparing bulk metallic samples and offers significant promise for the broader application of in situ liquid/gaseous cell S/TEM studies.

\section{ACKNOWLEDGMENTS}

Dr. A. Janssen and Dr. M.A. Kulzick are acknowledged for their insightful comments. S.S. acknowledges the Engineering and Physical Sciences Research Council UK PROMINENT program for support. In addition, this work was supported in part by research grants including grant nos. EP/G035954/1 and EP/J021172/1 and Defense Threat Reduction Agency grant no. HDTRA1-12-1-0013, the BP 2013 DRL Innovation Fund, as well as the Electron Microscopy Center in the Center for Nanoscale Materials, a US Department of Energy Office of Science User Facility under contract no. DE-AC02-06CH11357.

\section{References}

Abrams, I.M. \& McBain, J.W. (1944). A closed cell for electron microscopy. Science 100, 273-274.

Chee, S.W., Duquette, D.J., Ross, F.M. \& Hull, R. (2014). Metastable structures in $\mathrm{Al}$ thin films before the onset of corrosion pitting as observed using liquid cell transmission electron microscopy. Microsc Microanal 20(2), 1-7.

Dalili, N., Li, P., Kupsta, M., Liu, Q. \& Ivey, D.G. (2014). In situ TEM study of stability of TaRhx diffusion barriers using a novel sample preparation method. Micron 58, 25-31.

de Jonge, N., Peckys, D.B., Kremers, G.J. \& Piston, D.W. (2009). Electron microscopy of whole cells in liquid with nanometre resolution. Proc Natl Acad Sci U S A 106(7), 2159-2164.

Dens Solutions B.V. (2016). Available at http://denssolutions.com.

Evans, J.E., Jungjohann, K.L., Browning, N.D. \& Arslan, I. (2011). Controlled growth of nanoparticles from solution with in situ liquid transmission electron microscopy. Nano Lett 11(7), 2809-2813.

Evans, J.E., Jungjohann, K.L., Wong, P.C.K., Chiu, P.-L., Dutrow, G.H., Arslan, I. \& Browning, N.D. (2012). Visualizing macromolecular complexes with in situ liquid scanning transmission electron microscopy. Micron 43, 1085-1090.

Giannuzzi, L.A., Kempshall, B.W., Schwarz, S.M., Lomness, J.K., Prenitzer, B.I. \& Stevie, F.A. (2005). FIB lift-out specimen 
preparation techniques. In Introduction to Focused Ion Beams, Giannuzzi, L.A. \& Stevie, B.I. (Eds.), pp. 201-228. New York, NY: Springer.

GiannUZzi, L.A. \& STEvie, F.A. (1999). A review of focused ion beam milling techniques for TEM specimen preparation. Micron 30, 197-204.

Giannuzzi, L.A., Yu, Z., Yin, D., Harmer, M.P., Xu, Q., Smith, N.S., Chan, L., Hiller, J., Hess, D. \& Clark, T. (2015). Theory and new applications of ex situ lift out. Microsc Microanal 21(4), 1034-1048.

Holtz, M.E., Yu, Y., GaO, J., Abruña, H.D. \& Muller, D.A. (2013). In situ electron energy-loss spectroscopy in liquids. Microsc Microanal 19, 1027-1035.

HuANG, Z. (2004). Combining Ar ion milling with FIB lift-out technique for high quality site specific TEM samples. J Microsc 215, 219-223.

Hummingbird SCIENTific (2016). Available at http:// hummingbirdscientific.com.

Kestel, B. (1986). Polishing methods for metallic and ceramic transmission electron microscopy specimens, ANL-80-120/Rev.1 report, NTIS DE89016686 Issue Number 199005. Available at https://ntrl.ntis.gov/NTRL/dashboard/searchResults.xhtml?search Query $=$ ANL-80-120.

Mayer, J., Giannuzzi, L.A., Kamino, T. \& Michae, J. (2007). TEM sample preparation and FIB-induced damage. MRS Bull 32, 400-407.

Parent, L.R., Robinson, D.B., Cappillino, P.J., Hartnett, R.J., Abellan, P., Evans, J.E., Browning, N.D. \& Arslan, I. (2014). In situ observation of directed nanoparticle aggregation during the synthesis of ordered nanoporous metal in soft templates. Chem Mater 26(3), 1426-1433.

Parsons, D.F. (1974). Structure of wet specimens in electron microscopy: Improved environmental chambers make it possible to examine wet specimens easily. Science 186, 407-414.

Prestat, E., Smith, M., Jansen, A., Slater, T.J.A., Camargo, P.H.C., Kulzick, M.A., Burke, M.G., Haigh, S.J. \& Zaluzec, N.J. (2015). XEDS and EELS in the TEM at atmospheric pressure and high temperature. Microsc Microanal 21(Suppl 3), 247-248.

Proetto, M.T., Rush, A.M., et al. (2014). Dynamics of soft nanomaterials captured by transmission electron microscopy in liquid water. J Am Chem Soc 136(4), 1162-1165.

Protochips InC (2016). Available at http://www.protochips.com.

Radisic, A., Ross, F.M. \& Searson, P.C. (2006a). In situ study of the growth kinetics of individual island electrodeposition of copper. J Phys Chem B 110, 7862-7868.

Radisic, A., Vereecken, P.M., Searson, P.C. \& Ross, F.M. (2006b). The morphology and nucleation kinetics of copper islands during electrodeposition. Surf Sci 600, 1817-1826.
Schilling, S., Janssen, A., Burke, M.G., Zhong, X.L., Haigh, S.J., KulzicK, M.A. \& ZaluzeC, N.J. (2014). In situ analytical electron microscopy: Imaging and analysis of steel in liquid water, IT-7-O-2947. 18th International Microscopy Congress, Prague, September 2014, pp. 7-112.

Unocic, K.A., Allard, L.F., Coffey, D.W., More, K.L. \& Unocic, R.R. (2014). Novel method for precision controlled heating of TEM thin sections to study reaction processes. Microsc Microanal 20(Suppl 3), $1628-1629$.

Unocic, R., AdamczyK, L.A., Dudney, N.J., Alsem, D., Salmon, N.J. \& MoRE, K.L. (2011). In-situ TEM characterization of electrochemical processes in energy storage systems. Microsc Microanal 7(Suppl 2), 2011.

Williamson, M.J., Tromp, R.M., Vereecken, P.M., Hull, R. \& Ross, F.M. (2003). Dynamic microscopy of nanoscale cluster growth at the solid-liquid interface. Nat Mater 2(8), 532-536.

Young, R.J., Buxbaum, A. \& Peterson, B. (2008). Applications of insitu sample preparation and modeling of SEM-STEM imaging. Proceedings of the 34th International Symposium for Testing and Failure Analysis, Portland, OR, pp. 320-326.

Yuk, J.M., Park, J., Ercius, P., Kim, K., Hellebusch, J., Crommie, M.F., Lee, J.Y., Zettl, A. \& Alivisatos, A.P. (2012). High-resolution EM of colloidal nanocrystal growth using graphene liquid cells. Science 335(6077), 61-64.

Zaluzec, N.J., Burke, M.G., Haigh, S.J. \& Kulzick, M.A. (2014a). $\mathrm{X}$-ray energy-dispersive spectrometry during in situ liquid cell studies using an analytical electron microscope. Microsc Microanal 20, 323-329.

Zaluzec, N.J., Demortiere, A., Cook, R.E., Korttalia, R.E., Wen, J.G., Miller, D.J. \& KulZick, M.A. (2014b). X-ray and electron energy loss spectroscopy in liquids in the analytical S/TEM. Microsc Microanal 20(Suppl 3), 1518-1591.

Zheng, M., Claridge, S.A., Minor, A.M., Alivisatos, A.P. \& Dahmen, U. (2009). Nanocrystal diffusion in a liquid thin film observed by in situ transmission electron microscopy. Nano Lett 9, 2460-2465.

Zhong, X.L., Burke, M.G., Schilling, S., Haigh, S.J., Kulzick, M.A. \& ZALUZEC, N.J. (2014). Novel hybrid sample preparation method for in situ liquid cell TEM analysis. Microsc Microanal 20(Suppl 3), 口-

Ziemniak, S.E. \& Hanson, M. (2002). Corrosion behavior of 304 stainless steel in high temperature, hydrogenated water. Corros Sci 44(10), 2209-2230. 